\title{
Tat-interactive Protein-60KDA (TIP60) Regulates the Tumorigenesis of Lung Cancer In Vitro
}

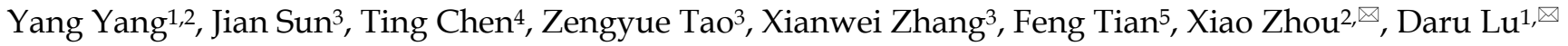 \\ 1. State Key Laboratory of Genetic Engineering and MOE Key Laboratory of Contemporary Anthropology, Institute of Genetics, School of Life Sciences, Fudan \\ University, Shanghai 200433, China; \\ 2. Department of Thoracic Surgery, Shanghai Pulmonary Hospital (Tongji University), Shanghai 200433, China; \\ 3. Department of General Surgery, the branch of Shanghai First People's Hospital, Shanghai 200081, China \\ 4. Department of Pharmacy, Xin-hua Hospital Affiliated to Medical School, Shanghai Jiao Tong University, Shanghai 200092, China \\ 5. Department of Pharmacy, the branch of Shanghai First People's Hospital, Shanghai 200081, China \\ $\triangle$ Corresponding authors: Dr. Daru Lu. Email: ludarufudan@sina.com; Tel: 86- 21-65115006; Fax: 86- 21-65115006. Postal address: Room C613, Life Science \\ Building, Jiangwan Campus of Fudan University, 2005 Songhu Rd., Shanghai, China, 200438 and Dr. Xiao Zhou. Email: zhouxiaosph@163.com; Tel: 86- \\ 21-65115006; Fax: 86- 21-65115006. Postal address: 507 Zhengmin Road, Shanghai, China, 200433 \\ (C) Ivyspring International Publisher. This is an open access article distributed under the terms of the Creative Commons Attribution (CC BY-NC) license \\ (https://creativecommons.org/licenses/by-nc/4.0/). See http://ivyspring.com/terms for full terms and conditions.
}

Received: 2017.02.15; Accepted: 2017.05.01; Published: 2017.07.20

\begin{abstract}
Histone acetyltransferases (HATs) play vital functions in the tumorigenesis of many solid organ malignancies. We previously screened a human HATs cDNA library and identified Tat-interactive protein-60KDa (TIP60) as a candidate critical HATs in the origination of lung cancer. In this study, our data suggested that overexpression of TIP60 inhibited the cell viability of A549 and H1299 cells since day 2. Compared to the control group, the viability of these two lung cancer cells was inhibited by $25 \%$ and $19 \%$ at day 6 with the overexpression of TIP60. It increased by $36 \%$ and $26 \%$ when TIP60 was knockdown for 6 days. The invasion ability of these two cells was also restrained when TIP60 was overexpressed. While knockdown of TIP60 had the opposite effect. Inhibition of TIP60 significantly promoted the expression of molecules in AKT signaling pathway especially c-Myc. Furthermore, compared to paired tumor-adjacent tissue, lung cancer tumors had low expression of TIP60. Therefore, we concluded that TIP60 might inhibit the viability and invasion ability of lung cancer cells through down-regulation of AKT signaling pathway.
\end{abstract}

Key words: TIP60; lung cancer; cell viability; AKT; Myc

\section{Introduction}

Despite advances made in early detection and improved treatments over the past ten years, cancer is still one of the leading causes of mortality around the world. Lung cancer ranks the highest in cancer lethality worldwide and is the most common newly diagnosed type in North America and Asia 1. Our previous study suggested that the smoking drive lung cancer decreased along with the implement of ban on smoking in public, while the genetic alterations related lung cancer rapidly increased ${ }^{2}$. Epigenetic aberration, including methylation and acetylation, was thought to catalyze the occurrence of genetic alterations in somatic cells and the communications between epigenetic and genetic alterations played pivotal role in cancer initiation and progression ${ }^{3}$.
Recently, more and more evidences on correlation between acetylation and tumorigenesis are emerging ${ }^{4-6}$. Acetylation is the reaction that introduces an acetyl functional group into the substrate. It is an important modification of proteins in cell biology. Several proteins, like histones, p53, and tubulins, have biological functions only after acetylation modification. This process requires the participation of histone acetyltransferases (HATs).

TIP60 (tat interacting protein $60 \mathrm{kD}$ ) is one of the most known HATs. It was involved in a vast variety of cellular processes (apoptosis, DNA damage repair, cell cycle progression and transcriptional regulation) $7-9$, and related to the occurrence of several tumors, like prostate, breast and colorectal cancer as well as 
lymphoma ${ }^{10-12}$. But whether TIP60 is an oncogene or tumor suppressor gene is still controversial ${ }^{13}$. In this study, we analyzed the correlation between TIP60 and lung cancer. We determined the effect of TIP60 on the cell viability and invasion changes of lung cancer cells and analyzed the expression changes of AKT signaling pathway caused by TIP60. Our data revealed important new insight into the relationship between TIP60 and lung cancer development that warrants further investigation of it as a novel cancer therapeutic target.

\section{Materials and methods}

\section{Ethical approval}

This study was approved by the Institutional Review Board (IRB) of Shanghai Pulmonary Hospital affiliated Tongji University. Written informed consents were obtained from all participants. The methods were carried out in accordance with the approved guidelines.

\section{Reagents and antibodies}

Lipofectamine 2000 (Life Technologies), 3-(4,5-dimethylthiazol-2-yl)-2,5-diphenyltetrazolium bromide (MTT) (Antgene), mouse antibodies against $\beta$-actin (Sigma); rabbit polyclonal antibodies against AKT, phospho-AKT, FoxO1, phospho-FoxO1, GSK3 $\beta$, phospho-GSK3 $\beta$, cMyc and TIP60 (CST) were purchased from the indicated manufacturers.

\section{Cell culture and plasmid construction}

Human lung cancer A549 and H1299 cells were obtained from ATCC and maintained in DMEM medium with the supplement of $10 \%$ FBS. The TIP60 and TIP60-RNAi was constructed the same with the previous. The knockdown efficient of TIP60-RNAi was determined by the immunoblotting analysis.

\section{The stable TIP60 expression/knockdown A549 and $\mathrm{H} 1299$ cells}

The HEK293T cells were used as the retrovirus packaging cells. The packaging plasmids (pGAG-Pol and pVSV-G) together with TIP60 or TIP60-RNAi retroviral plasmid were transfected into HEK293T cells for $24 \mathrm{~h}$. Then the cells were incubated with new medium without antibiotics for another $24 \mathrm{~h}$. The cell culture medium which was collected and filtered with 0.22 um filter was added to A549 and H1299 cells with polybrene $(10 \mathrm{mg} / \mathrm{ml})$. The infected cells were selected with puromycin $(0.5 \mathrm{mg} / \mathrm{ml})$ for 7 days before additional experiments.

\section{Cell viability assay}

The stable TIP60 expression or knockdown A549 and H1299 cells (2X103) were seeded on the 96 well plates. Then it was determined by 3- (4,5-dimethylthi- azol-2-yl)-2,5-diphenyltetrazolium bromide (MTT) at day 2, 4 and 6. The MTT was added to each well, and incubated for $4 \mathrm{hr}$. Dimethyl sulfoxide $(100 \mu \mathrm{l})$ was then added to the cells for $30 \mathrm{~min}$. The absorbance was measured at $570 \mathrm{~nm}$.

\section{Cell invasion assay}

The 12-well transwell plates $(8.0 \mu \mathrm{m}$ pore filters) (Corning, NY) were used to analyze the cell invasion ability. The protocol followed the recommendation of manufacturers. Briefly, the upper chamber of each transwell plate was coated with matrigel basement membrane matrix and seeded with $2 \times 10^{5}$ lung cancer cells in serum-free DMEM medium. While the bottom chamber was filled with DMEM medium including $10 \%$ FBS. The whole plate was incubated for 1 day at regular conditions. Then the transwell membrane was fixed, stained and photographed. The penetrated cells were calculated with the ImageJ software.

\section{Immunoblotting Analysis}

Cells from $10 \mathrm{~cm}$ cell dish were lysed in $1 \mathrm{ml}$ Nonidet P-40 lysis buffer $(20 \mathrm{mM}$ Tris- $\mathrm{HCl}, \mathrm{PH}$ 7.4-7.5, $150 \mathrm{mM} \mathrm{NaCl}, 1 \mathrm{mM}$ EDTA, $1 \%$ Nonidet P-40, $10 \mu \mathrm{g} / \mathrm{mL}$ aprotinin, $10 \mu \mathrm{g} / \mathrm{mL}$ leupeptin, and $1 \mathrm{mM}$ phenylmethylsulfonyl fluoride). The detected molecules were fractionated by SDS/PAGE eletro phoresis and subsequent immunoblotting analysis was performed with the indicated antibody.

\section{Patients and specimen collection}

Consecutive primary lung cancer patients who were admitted into the Shanghai Pulmonary Hospital affiliated Tongji University from May. 2016 to Sep. 2016 were recruited. None of them received any anticancer therapies before surgery. The diagnosis was confirmed by pathologically test and radical surgical resection. Fresh primary tumor specimens which contained more than $50 \%$ tumor cells were collected during the surgery and the total protein was isolated immediately. The expression of TIP60 was analyzed by immunoblotting analysis with the antibody to TIP60.

\section{Statistical analysis}

All data were analyzed by the SPSS package for Windows (Version 18.0, Chicago, IL). $\chi 2$ test was used to analyze the differences. P value $<0.05$ was considered statistically significant.

\section{Results}

\section{TIP60 regulates the viability of lung cancer cells}

In order to analyze the role of TIP60 in tumorgenesis of lung cancer, we observed the effect of TIP60 on cell viability of lung cancer cells. Over- 
expression of TIP60 significantly inhibited the proliferation of A549 and H1299 cells since day 2. Compared to the control group, the growth of these two lung cancer cells was inhibited by $25 \%$ and $19 \%$ at day 6. Knockdown of TIP60 had the opposite effect. The number of cells increased by $36 \%$ and $26 \%$ when TIP60 was knockdown for 6 days (Fig 1).

\section{The invasion ability of lung cancer cells is affected by TIP60}

After determining the role of TIP60 in regulating cell viability, we observed the changes of invasion ability caused by TIP60. The number of cells crossing through the transwell membrane decreased with the overexpression of TIP60. While knockdown of TIP60 promoted more lung cancer cells going through the membrane. Both A549 cells and H1299 cells had similar results (Fig 2).

\section{TIP60 is involved in AKT signaling pathway}

In order to analyze the molecular mechanism on
TIP60 regulating lung cancer, we further studied the expression changes of downstream molecules of TIP60 with the knockdown of TIP60. The inhibition of TIP60 significantly promoted the expression of molecules in AKT signaling pathway. AKT was activated as the phosphorylation at threonine 308 (Thr308) and serine 473 (Ser473) promoted. The phosphorylation of GSK3 $\beta$ and FOXO1was also promoted. Furthermore, the expression of cMyc significantly increased (Fig 3).

\section{TIP60 decreased in lung cancer tumors}

To further study the role of TIP60 in lung cancer, we examined its clinical relevance in cancer patients. A total of 18 lung cancer patients were recruited in this study. Most of them are Han people. TIP60 exhibited lower expression state in cancer tissues than that in paired tumor-adjacent tissues in $13(72.22 \%)$ patients (Fig 4).
A

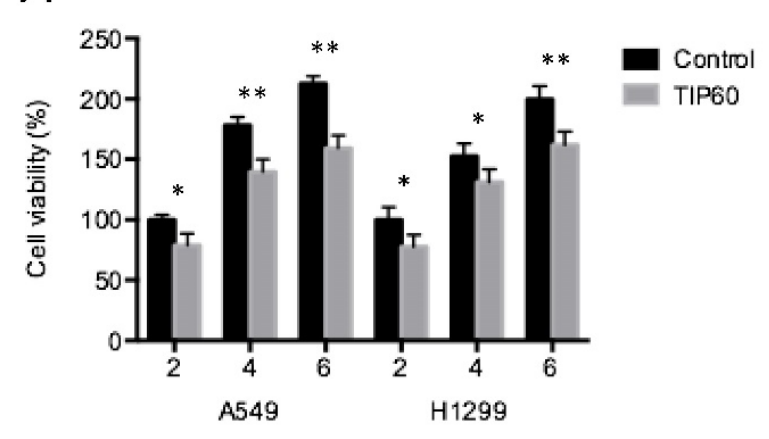

B

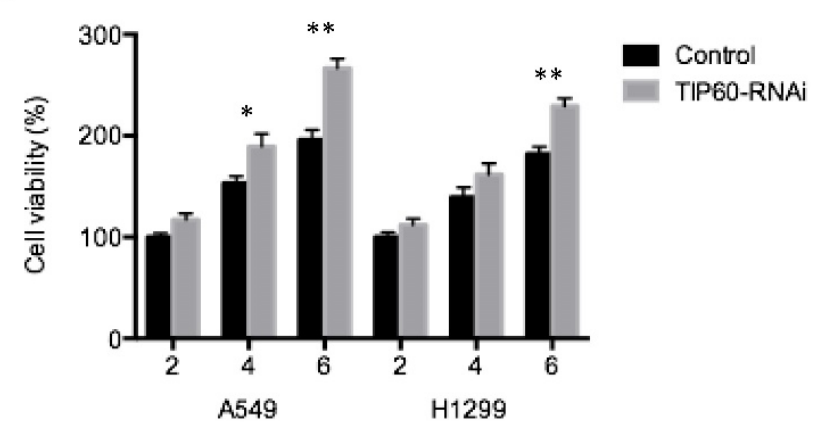

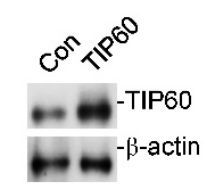

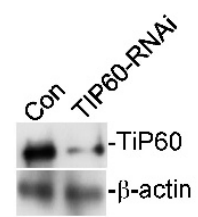

Figure 1. TIP60 regulates the viability of A549 and H1299 cells. (A) Overexpression of TIP60 inhibited the cell viability. (B) Knockdown of TIP60 promoted the cell viability since day 2. The efficient of TIP60 overexpression and inhibition was shown at the bottom of each figure. *: $\mathrm{P}<0.05, * *: \mathrm{P}<0.01$.

A

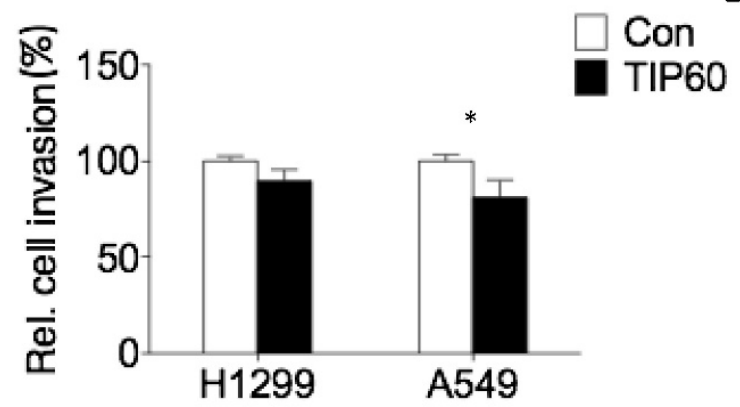

B

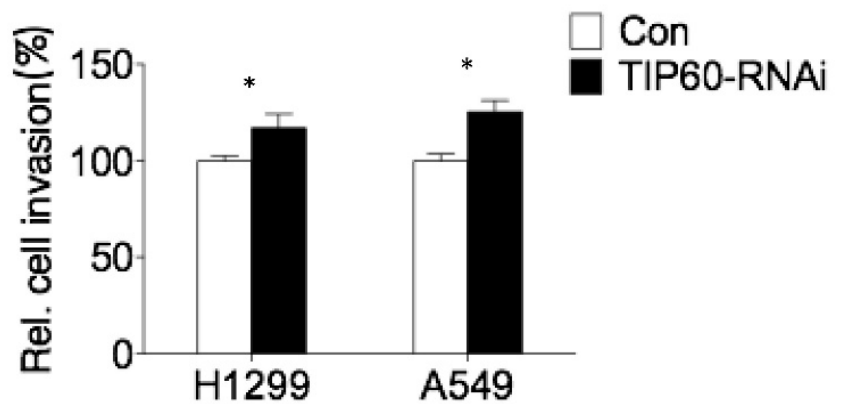

Figure 2. TIP60 regulates the invasion ability of A549 and H1299 cells. (A) Overexpression of TIP60 inhibited the invasion of these two lung cancer cells. (B) Knockdown of TIP60 promoted the cell invasion. *: $\mathrm{P}<0.05$. 


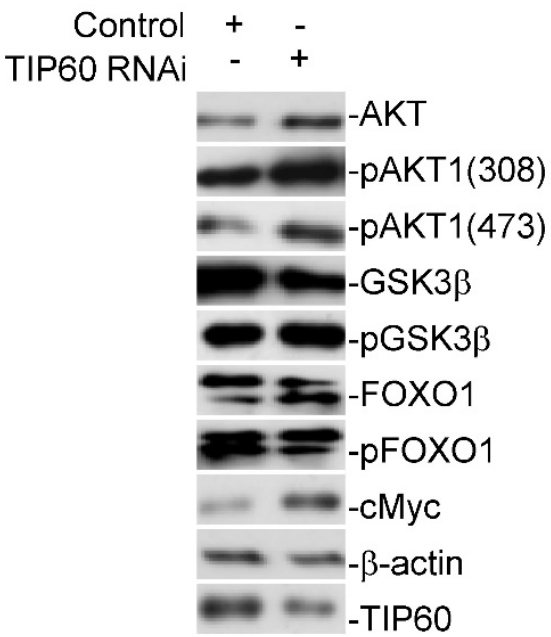

Figure 3. TIP60 is participated in the AKT signaling pathway.

\section{TIP60}

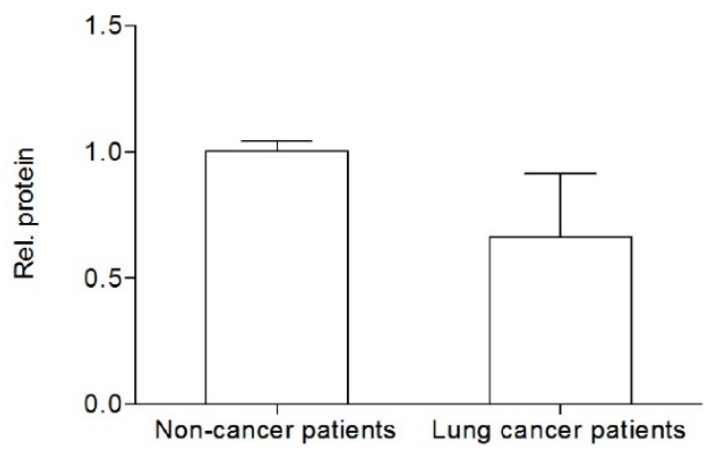

Figure 4. TIP60 under-expresses in lung cancer tumors.

\section{Discussion}

TIP60 is a subunit of the evolutionarily conserved NuA4 complex ${ }^{14}$. TIP60 was thought to be close with transcription regulation because several of its substrates were transcript factors. It has wide range of substrate, including histones (H4 at lysines 5, 8, 12 and 16, H2A, H2A.X and H2A.Z) ${ }^{15}$ and non-histones (p53, Notch1 and androgen receptor) 7,16-17. It was related to several tumors through regulating the stability or activity of its substrate. Our results contributed to support the conclusion that TIP60 was a tumor suppressor. It inhibited the cell viability and invasion of lung cancer cells and under-expressed in lung cancer patients. The mechanism of TIP60 inhibitting tumorigenesis might be: first, TIP60 positively response to the activation of the oncogeneinduced DNA damage response pathway; second, the activity of AKT signaling pathway increased along with the under-expression of TIP60. Our results suggested that the symbol of AKT activation, the phosphorylation at Thr308 and Ser473 was promoted when TIP60 was knockdown. The oncoprotein, cMyc, was also overexpressed. Furthermore, TIP60 was also involved in many other anti-proliferative processes, such as p53 activation, and apoptosis ${ }^{7}$. We believed that the suppression of tumor was the result of multiple biological function.

The impressive progresses made in tumor biology help us understand that each tumor is unique and the traditional strategy of applying the same drug to different patients needs improvement. The biological profile of tumor provides the new strategy, precision medicine, to develop treatment plan which meets the urgent need to alleviate the growing cancer burden. In the past ten years, part of patients with breast and lung cancer already benefited from the personalized treatment which aimed to treat patients according to their own medical history, physiological status, and molecular characteristics.

Our results confirmed the correlation between TIP60 and lung cancer and suggested it might represent a novel candidate target for the intervention. TIP60 is a member of MYST acetyltransferases family which had the distinct conserved histone acetyltransferase domain. The unique MYST domain is composed of three parts: a C2HC-type zinc finger in $\mathrm{N}$ - terminal, a central region associated with acetyl-CoA cofactor binding, a helix- turn-helix DNA-binding motif in C-terminal ${ }^{18}$. The special characteristic features might can be used to develop the treatment target.

In conclusion, our data establish the role for TIP60 in the tumorigenesis of lung cancer. Of clinical relevance is the fact that our results may contribute to develop the new molecule treatment targets for lung cancer.

\section{Acknowledgements}

This work was supported by grants from the National Natural Scientific Foundation of China (81602412), Scientific research project of Shanghai Municipal Commission of Health and Family Planning (20164Y0121), the Shanghai Science and Technology Commission Grant (No. 15411965400), Medical research foundation of Shanghai Hongkou district health and family planning commission (HW1302-09), Shanghai university scientific selection and cultivation for outstanding young teachers in special fund (zzjdyx13087). The originator of the funds had no role or impact in the design of the study.

\section{Competing Interests}

None.

\section{References}

1. World Health Organization. Global battle against cancer won't be won with treatment alone--effective prevention measures urgently needed to prevent cancer crisis. Cent Eur J Public Health 2014;22(1):23-28. 
2. Yang $Y$, Shi C, Sun $H$, et al. Elderly male smokers with right lung tumors are viable candidates for KRAS mutation screening. Sci Rep. 2016;6:18566.

3. You JS, Jones PA. Cancer genetics and epigenetics: two sides of the same coin? Cancer Cell. 2012; 22(1): 9-20.

4. Yen CY, Huang HW, Shu CW, et al. DNA methylation, histone acetylation and methylation of epigenetic modifications as a therapeutic approach for cancers. Cancer Lett. 2016;373(2):185-192.

5. Shukla S, Sharma A, Pandey VK, et al. Concurrent acetylation of FoxO1/3a and p53 due to sirtuins inhibition elicit Bim/PUMA mediated mitochondrial dysfunction and apoptosis in berberine-treated HepG2 cells. Toxicol Appl Pharmacol. 2016;291(1):70-83.

6. Kato Y, Egusa C, Maeda T, et al. Combination of retinoid and histone deacetylase inhibitor produced an anti-tumor effect in cutaneous T-cell lymphoma by restoring tumor suppressor gene, retinoic acid receptor $\beta 2$, via histone acetylation. J Dermatol Sci. 2016;81(1):17-25.

7. Xu Y, Liao R, Li N, et al. Phosphorylation of Tip60 by p38a regulates p53-mediated PUMA induction and apoptosis in response to DNA damage. Oncotarget. 2014;5(24):12555-12572.

8. Bassi C, Li YT, Khu K, et al. The acetyltransferase Tip60 contributes to mammary tumorigenesis by modulating DNA repair. Cell Death Differ. 2016;23(7):1198-1208.

9. Jang SM, Kim JW, Kim CH, et al. KAT5-mediated SOX4 acetylation orchestrates chromatin remodeling during myoblast differentiation. Cell Death Dis 2015;6:e1857.

10. Pandey AK, Zhang Y, Zhang S, et al. TIP60-miR-22 axis as a prognostic marker of breast cancer progression. Oncotarget. 2015;6(38):41290-41306.

11. Mattera L, Escaffit F, Pillaire MJ, et al. The p400/Tip60 ratio is critical for colorectal cancer cell proliferation through DNA damage response pathways. Oncogene. 2009;28(12):1506-1517.

12. Litvinov IV, Netchiporouk E, Cordeiro B, et al. Ectopic expression of embryonic stem cell and other developmental genes in cutaneous T-cell lymphoma. Oncoimmunology. 2014;3(11):e970025.

13. Judes G, Rifaï K, Ngollo M, et al. A bivalent role of TIP60 histone acetyl transferase in human cancer. Epigenomics. 2015;7(8):1351-1363.

14. Ravens S, Yu C, Ye T, et al. Tip60 complex binds to active Pol II promoters and a subset of enhancers and co-regulates the c-Myc network in mouse embryonic stem cells. Epigenetics Chromatin. 2015;8:45.

15. Grézy A, Chevillard-Briet $M$, Trouche $D$, et al. Control of genetic stability by a new heterochromatin compaction pathway involving the Tip60 histone acetyltransferase. Mol Biol Cell. 2016;27(4):599-607.

16. Kim MY, Ann EJ, Kim JY, et al. Tip60 histone acetyltransferase acts as a negative regulator of Notch1 signaling by means of acetylation. Mol Cell Biol. 2007;27(18):6506-6519.

17. Jaganathan A, Chaurasia P, Xiao GQ, et al. Coactivator MYST1 regulates nuclear factor- $\mathrm{kB}$ and androgen receptor functions during proliferation of prostate cancer cells. Mol Endocrinol. 2014;28(6):872-885.

18. Yuan H, Rossetto D, Mellert H, et al. MYST protein acetyltransferase activity requires active site lysine autoacetylation. EMBO J. 2012;31(1):58-70. 